\title{
Magnetic fields in nearby galaxies: prospects with future radio telescopes
}

\author{
Rainer Beck* \\ Max-Planck-Institut für Radioastronomie, Auf dem Hügel 69, 53121 Bonn, Germany \\ E-mail: rbeck@mpifr-bonn.mpg • de
}

\begin{abstract}
The origin of magnetic fields in the Universe is an open problem in astrophysics and fundamental physics. Our present-day knowledge is limited to regions of strong magnetic fields and to starforming disks of galaxies. Cosmic-ray electrons emitting at high $(\mathrm{GHz})$ radio frequencies can propagate only a few kpc from their places of origin due to their lifetime limited by energy losses. Low-energy electrons emitting at low frequencies suffer less from energy losses and can propagate further into the intergalactic medium. The prospects are threefold: Firstly, LOFAR will map the structure of weak magnetic fields in the outer regions and halos of galaxies and in the Milky Way. Polarized emission is an excellent tracer of past interactions with other galaxies and with the interstellar medium. Secondly, high-resolution polarization observations are needed at high frequencies with the EVLA and SKA to trace the structure of magnetic fields in the disks and central regions of galaxies in unprecedented detail. The SKA can also detect polarized emission from distant, unresolved galaxies. Thirdly, Faraday rotation measures (RM) are signatures of regular magnetic fields generated by the dynamo mechanism. All-sky surveys of Faraday rotation measures (RM) towards polarized background sources will be used to model the structure and strength of the regular magnetic fields in the Milky Way, the interstellar medium of galaxies and the intergalactic medium. The novel method of RM Synthesis, applied to spectro-polarimetric data cubes, is able to separate RM components from different distances and may allow 3-D Faraday tomography. This will open a new era in the observation of cosmic magnetic fields. "Key Science" Projects on cosmic magnetism are organized for the Low Frequency Array (LOFAR), the planned Square Kilometre Array (SKA) and the Australian SKA Pathfinder telescope (ASKAP).
\end{abstract}

Panoramic Radio Astronomy: Wide-field 1-2 GHz research on galaxy evolution - PRA2009

June 02 - 052009

Groningen, the Netherlands

\footnotetext{
* Speaker.
} 


\section{Origin of galactic magnetic fields}

The origin of the first magnetic fields in the Universe is still a mystery [35]. A large-scale primordial field is hard to maintain in a young galaxy because the galaxy rotates differentially, so that field lines get strongly wound up during galaxy evolution, while observations show significant pitch angles. Turbulent "seed" fields in young galaxies can originate from the Weibel instability in shocks during the cosmological structure formation [22] or can be injected by the first stars or jets generated by the first black holes [27]. In any case a mechanism to sustain and organize the magnetic field is required.

The most promising mechanism is the dynamo $[6,8]$ which transfers mechanical into magnetic energy. In young galaxies without ordered rotation a small-scale dynamo [8] probably amplified the seed fields from the protogalactic phase to the energy density level of turbulence within less than $10^{9} \mathrm{yr}[1]$ (see also this volume). To explain the generation of large-scale fields, the mean-field $\alpha \Omega$-dynamo has been developed as a simplified model. It needs turbulence, differential rotation and helical gas flows ( $\alpha$ effect), generated by supernova explosions [11, 15] or cosmic-ray driven Parker loops [16, 24, 26]. The mean-field $\alpha \Omega$-dynamo in galaxy disks predicts that within a few $10^{9} \mathrm{yr}$ large-scale regular fields are excited from the seed fields, described as "modes" with different azimuthal symmetry in the disk and vertical symmetry perpendicular to the disk plane $[1,6]$.

The mean-field dynamo generates large-scale helicity with a non-zero mean in each hemisphere. As total helicity is a conserved quantity, the dynamo is quenched by the small-scale fields with opposite helicity unless these are removed from the system [29]. It seems that outflows are essential for an effective mean-field dynamo.

Classical dynamo models are strongly simplified because small and large scales are artificially separated and the back-reaction of the field onto the gas flow is not included. Dynamical MHD models of rotating galaxy disks where dynamo action at all scales emerges automatically are just becoming feasible with present-day computers [17].

\section{Present-day knowledge about galactic magnetic fields}

The typical average "equipartition" strength of the total magnetic field in spiral galaxies is about $10 \mu \mathrm{G}$, assuming energy equipartition between cosmic rays and magnetic fields. Radiofaint, gas-poor galaxies like M 31 have weaker total magnetic fields (about $5 \mu \mathrm{G}$ on average, higher in star-forming regions), while gas-rich spiral galaxies with high star-formation rates, like M 51 (Fig. 1) and NGC 6946, have total field strengths of $15 \mu \mathrm{G}$ on average and 20-30 $\mu \mathrm{G}$ in their spiral arms. The mean energy density of the equipartition magnetic field (and hence that of the cosmic rays) is $\simeq 10^{-11} \mathrm{erg} \mathrm{cm}^{-3}$ in NGC 6946 and $\simeq 10^{-12} \mathrm{erg} \mathrm{cm}^{-3}$ in M 33 [3, 32], about 10 times larger than that of the ionized gas, but similar to that of the turbulent gas motions across the whole star-forming disk.

The strongest total fields of $50-100 \mu \mathrm{G}$ were found so far in starburst galaxies, like M 82 and the "Antennae" NGC 4038/9 [10], and in nuclear starburst regions, like in the centers of NGC 1097 and other barred galaxies [7]. In starburst galaxies the equipartition field strength is probably underestimated due to strong energy losses of the cosmic rays [33] which was recently confirmed by Zeeman measurements of $\mathrm{OH}$ maser lines [28]. 


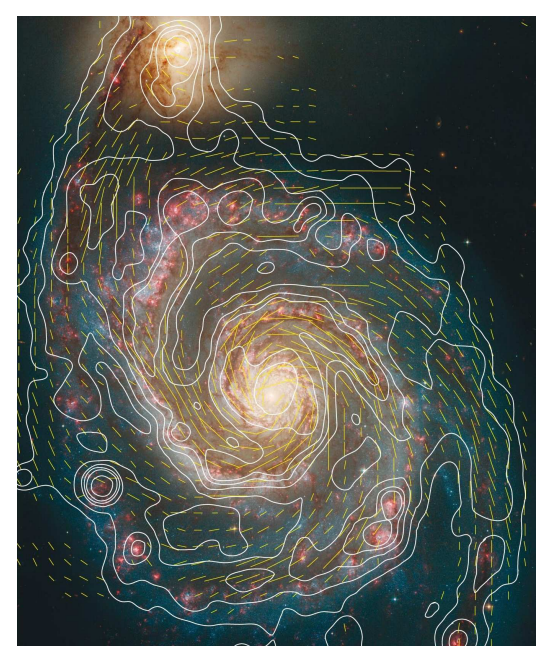

Figure 1: Total radio emission (contours) and $B-$ vectors of M 51 observed at $6 \mathrm{~cm}$ wavelength with the VLA and Effelsberg telescopes and smoothed to 15 " resolution [13], overlaid onto an optical image from the HST (Copyright: MPIfR Bonn and Hubble Heritage Team. Graphics: Sterne und Weltraum).

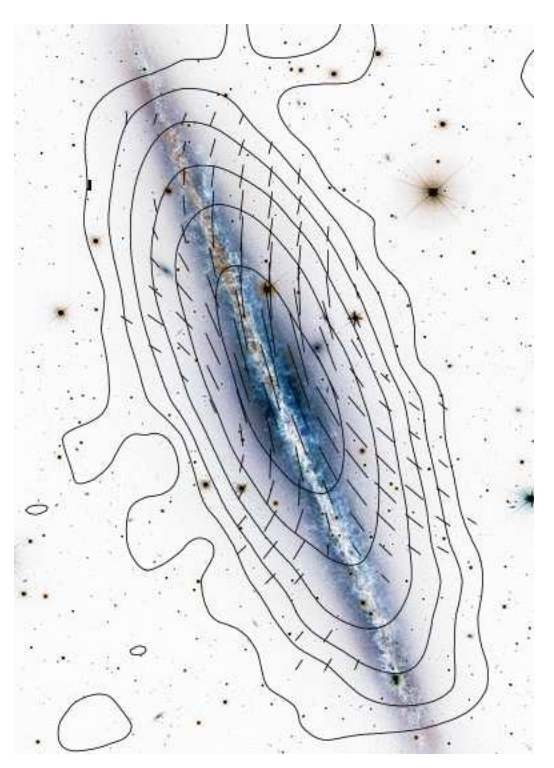

Figure 2: Total radio emission (84" resolution) and $B$-vectors of the edge-on spiral galaxy NGC 891, observed at $3.6 \mathrm{~cm}$ wavelength with the Effelsberg 100m telescope [21]. The background optical image is from the CFHT (Copyright: MPIfR Bonn and CFHT/Coelum).

The degree of radio polarization within the spiral arms is only a few \%; hence the field in the spiral arms is mostly tangled or randomly oriented within the telescope beam, the width of which corresponds to scales between a few $100 \mathrm{pc}$ and a few kpc. Turbulent fields in spiral arms are probably generated by turbulent gas motions related to star formation activity.

The ordered (regular and/or anisotropic) fields traced by the polarized synchrotron emission are generally strongest $(10-15 \mu \mathrm{G})$ in the regions between the optical spiral arms and oriented parallel to the adjacent spiral arms, in some galaxies forming magnetic arms. These are probably generated by the mean-field dynamo [6]. However, in galaxies with strong density waves like M 51 (Fig. 1) the turbulent field is compressed at the inner edge of the spiral arms and becomes anisotropic, while the regular dynamo field is weak [13]. The ordered magnetic field forms spiral patterns in almost every galaxy [2] and also in the central regions of galaxies and circum-nuclear gas rings [7]. Spiral fields can be generated by compression at the inner edge of spiral arms, by shear in interarm regions, or by dynamo action [6].

Large-scale patterns of Faraday rotation measures (RM) are signatures of regular dynamo fields and can be inferred from polarized emission of the galaxy disks [20] or from RM data of polarized background sources [30]. The Andromeda galaxy M 31 hosts a dominating axisymmetric disk field [12], as predicted by dynamo models. However, in many observed galaxy disks no clear patterns of Faraday rotation were found. Either several dynamo modes are superimposed and 
cannot be distinguished with the limited sensitivity and resolution of present-day telescopes, or the timescale for the generation of large-scale modes is longer than the galaxy's lifetime, or the regular field was distorted by interaction or a major merger [1].

Nearby galaxies seen "edge-on" generally show a disk-parallel field near the disk plane. Highsensitivity observations of edge-on galaxies like NGC 891 (Fig. 2) and NGC 253 [19] revealed vertical field components in the halo forming an X-shaped pattern. The field is probably transported from the disk into the halo by a gas outflow emerging from the disk.

\section{Limitations of observations with present-day radio telescopes}

If the magnetic field is dynamically important, dynamo MHD models are needed that include the back-reaction onto the gas flow. Detailed comparisons with observations of the field structure and the distributions and velocities of the various gas components need spatial resolutions of better than 100 pc. Present-day radio telescopes do not provide sufficient sensitivity at such resolutions because the observed signal from extended sources decreases with the beam area.

The observation of synchrotron emission from galaxies only reveals magnetic fields illuminated by cosmic-ray electrons (CRE) accelerated by supernova shock fronts in regions of strong star formation. As the diffusion length of CRE is limited by the Alfvén speed and the lifetime due to synchrotron and inverse Compton losses, radio images at centimeter wavelengths (synchrotron + thermal) are similar to images of star-forming regions as observed in the far-infrared. Radio emission from the outer disks and halos of galaxies is very weak due to the lack of emitting electrons. Hence, the strength and extent of magnetic fields into the intergalactic space is unknown.

Asymmetric distributions of the polarized emission from galaxies in the Virgo cluster show that the outer magnetic fields have been strongly compressed [34]. Polarized emission is an excellent tracer of past interactions between galaxies or with the intergalactic medium, but the observation is still difficult.

Faraday rotation is a signature of regular fields as generated by dynamo action, but the existing data are still scarce and often do not reveal clear patterns. Higher angular resolution is needed to distinguish between a spectrum of superimposed dynamo modes and anisotropic (sheared) fields. Most of the existing RM data also suffer from low spectral resolution causing depolarization by different Faraday rotation components within the beam or along the line of sight. RM Synthesis is capable to reveal such multiple RM components but needs a large number of channels over a wide frequency range $[9,18]$.

RM data from pulsars in the Milky Way indicate several field reversals at various Galactic radii [25]. However, no model of a large-scale field structure is statistically safe [23]. Similar to external galaxies, the Milky Way's regular field probably has a complex structure that cannot be resolved with the existing number of pulsar RM.

\section{Prospects}

Future radio telescopes will widen the range of observable magnetic phenomena. Highresolution, deep observations at high frequencies, where Faraday effects are small, require a major increase in sensitivity for continuum observations which will be achieved by the Extended Very 
Large Array (EVLA) and the planned Square Kilometre Array (SKA). We wish to resolve the detailed structure of the ISM and halo fields and distinguish sheared loops from regular fields with dynamo-type patterns [30]. The SKA will also allow to measure the Zeeman effect in much weaker magnetic fields in the Milky Way and in nearby galaxies.

Forthcoming low-frequency radio telescopes like the Low Frequency Array (LOFAR), Murchison Widefield Array (MWA), Long Wavelength Array (LWA) and the low-frequency SKA will be suitable instruments to search for extended synchrotron radiation at the lowest possible levels in outer galaxy disks and clusters and the transition to intergalactic space [4]. The detection of radio emission from the intergalactic medium would allow us to probe the existence of magnetic fields in such rarified regions, measure their intensity, and investigate their origin and their relation to the structure formation in the early Universe.

Nearby galaxies seen edge-on generally show a disk-parallel field near the disk plane, so that polarized emission can also be detected from unresolved galaxies [31] (see also this volume). With the SKA ordered fields in young galaxies can be searched for.

Radio spectro-polarimetric observations in many narrow frequency channels allows application of RM Synthesis [9, 18]. Faraday depolarization occuring in wide frequency bands is reduced and features at different distances along the line of sight can be separated. If the medium has a relatively simple structure, for example a few emitting regions and Faraday screens, Faraday tomography will become possible. This method is going to revolutionize radio polarization observations.

A reliable model for the global structure of the magnetic field of the Milky Way and nearby galaxies needs a much higher number of pulsar and extragalactic RM, hence larger sensitivity and/or higher survey speed. The POSSUM-wide survey at $1.4 \mathrm{GHz}$ with the planned Australia SKA Pathfinder (ASKAP) telescope will measure about 100 RM from polarized extragalactic sources per square degree. With $12 \mathrm{~h}$ integration at $1.4 \mathrm{GHz}$, the SKA will be able to detect $1 \mu \mathrm{Jy}$ sources and measure about 10.000 RM per square degree. The SKA "Magnetism" Key Science Project plans to observe an all-sky RM grid with $1 \mathrm{~h}$ integration per field which should contain about $10^{4} \mathrm{RM}$ values from pulsars with a mean spacing of $\simeq 30^{\prime}$ and about $10^{8} \mathrm{RM}$ from compact polarized extragalactic sources at a mean spacing of just $\simeq 1.5^{\prime}$ [14]. This survey will be used to model the structure and strength of the magnetic fields in the foreground of the Milky Way, in intervening galaxies, and in the intergalactic medium [5]. $10 \mathrm{RM}$ values in the solid angle area of a foreground object are already sufficient to recognize a simple large-scale field structure, while more than 1000 values are required for a detailed field reconstruction [30]. As the accuracy depends on the polarized flux of the background source, the distance range of this method is much larger than by direct imaging of the polarized emission from the intervening galaxy. Looking back into time, the future telescopes can shed light on the origin and evolution of cosmic magnetic fields.

\section{References}

[1] T. G. Arshakian, R. Beck, M. Krause and D. Sokoloff, A\&A 494, 21 (2009).

[2] R. Beck, in Cosmic Magnetic Fields, eds. R. Wielebinski and R. Beck, Springer, Berlin, 2005, p. 41.

[3] R. Beck, $A \& A$ 470, 539 (2007). 
[4] R. Beck, Rev. Mexicana AyA 36, 1 (2009).

[5] R. Beck and B. M. Gaensler, in Science with the Square Kilometer Array, eds. C. Carilli and S. Rawlings, New Astr. Rev. 48, Elsevier, Amsterdam, 2004, p. 1289.

[6] R. Beck, A. Brandenburg, D. Moss, A. Shukurov and D. Sokoloff, Ann. Rev. A\&A 34, 155 (1996).

[7] R. Beck, A. Fletcher, A. Shukurov et al., $A \& A$ 444, 739 (2005).

[8] A. Brandenburg and K. Subramanian, Phys. Rep. 417, 1 (2005).

[9] M. A. Brentjens and A. G. de Bruyn, $A \& A$ 441, 1217 (2005).

[10] K. T. Chyży and R. Beck, $A \& A$ 417, 541 (2004).

[11] K. Ferrière and D. Schmitt, $A \& A$ 358, 125 (2000).

[12] A. Fletcher, E. M. Berkhuijsen, R. Beck and A. Shukurov, $A \& A$ 414, 53 (2004).

[13] A. Fletcher, R. Beck, A. Shukurov, E. M. Berkhuijsen and C. Horellou, MNRAS, in press (2010).

[14] B. M. Gaensler, R. Beck and L. Feretti, in Science with the Square Kilometer Array, eds. C. Carilli and S. Rawlings, New Astr. Rev. 48, Elsevier, Amsterdam, 2004, p. 1003.

[15] O. Gressel, D. Elstner, U. Ziegler and G. Rüdiger, A\&A 486, L35 (2008).

[16] M. Hanasz, G. Kowal, K. Otmianowska-Mazur and H. Lesch, ApJ 605, L33 (2004).

[17] M. Hanasz, D. Wóltański, K. Kowalik and R. Pawlaszek, in Cosmic Magnetic Fields: From Planets, to Stars and Galaxies, eds. K. G. Strassmeier, A. G. Kosovichev and J. E. Beckman, Cambridge Univ. Press, Cambridge, 2009, p. 549.

[18] G. Heald, R. Braun and R. Edmonds, A\&A 503, 409 (2009).

[19] V. Heesen, M. Krause, R. Beck and R.-J. Dettmar, $A \& A$ 506, 1123 (2009).

[20] M. Krause, in Galactic and Intergalactic Magnetic Fields, eds. R. Beck, R. Wielebinski and P. P. Kronberg, Kluwer, Dordrecht, 1990, pp. 187.

[21] M. Krause, Rev. Mex. AyA 36, 25 (2009).

[22] M. Lazar, R. Schlickeiser, R. Wielebinski and S. Poedts, ApJ 693, 1133 (2009).

[23] H. Men, K. Ferrière and J. L. Han, $A \& A$ 486, 819 (2008).

[24] D. Moss, A. Shukurov and D. Sokoloff, $A \& A$ 343, 120 (1999).

[25] A. Noutsos, in Cosmic Magnetic Fields: From Planets, to Stars and Galaxies, eds. K. G. Strassmeier, A. G. Kosovichev and J. E. Beckman, Cambridge Univ. Press, Cambridge, 2009, p. 15.

[26] E. N. Parker, ApJ 401, 137 (1992).

[27] M. J. Rees, in Cosmic Magnetic Fields, eds. R. Wielebinski and R. Beck, Springer, Berlin, 2005, p. 1.

[28] T. Robishaw, E. Quataert and C. Heiles, ApJ 680, 981 (2008).

[29] A. Shukurov, D. Sokoloff, K. Subramanian and A. Brandenburg, A\&A 448, L33 (2006).

[30] R. Stepanov, T. G. Arshakian, R. Beck, P. Frick and M. Krause, $A \& A$ 480, 45 (2008).

[31] J. M. Stil, M. Krause, R. Beck and A. R. Taylor, ApJ 693, 1392 (2009).

[32] F. Tabatabaei, M. Krause, A. Fletcher and R. Beck, A\&A 490, 1005 (2008).

[33] T. A. Thompson, E. Quataert, E. Waxman, N. Murray and C. L. Martin, ApJ 645, 186 (2006).

[34] B. Vollmer, M. Soida, R. Beck et al., A\&A 464, L37 (2007).

[35] L. M. Widrow, Rev. Mod. Phys. 74, 775 (2002). 